\title{
Biofilm physical structure, internal diffusivity and tortuosity
}

\author{
L.F. Melo \\ Laboratory for Process, Environmental and Energy Engineering, Department of Chemical Engineering, \\ Faculty of Engineering of the University of Porto, Rua Roberto Frias, 4200-465 Porto, Portugal \\ (E-mail:Imelo@fe.up.pt)
}

\begin{abstract}
The paper proposes tortuosity as a physical concept particularly useful to interpret internal diffusivities in terms of biofilm structure. Results from different authors are presented showing how average effective diffusivities in biofilms (measured with inert tracers) vary with the fluid velocity: in the case of biofilms formed under turbulent flow conditions, an increase in fluid velocity corresponds to a decrease in the diffusivity, although sometimes this decrease is very slight; however, in laminar flow situations, no common trend is found from research group to research group.

Keywords Biofilms; internal diffusivity; tortuosity factor; velocity effects
\end{abstract}

\section{Introduction}

Typically, in biofilm systems substrates are transported across the liquid to the biofilmwater interface, then move on through the biofilm matrix and are finally consumed by the microorganisms living inside the biolayer. Mass transfer and biological reaction can then be considered to be consecutive phenomena. In such cases, the slowest mechanism will be the "limiting step" and will control the overall rate of substrate consumption and biological activity. Since internal mass transfer is frequently the slowest step, this inevitably makes it an important target for biofilm research.

Particularly relevant is the bidirectional process by which the biofilm physical structure (i.e. the spatial arrangement and relative concentration of dry matter and water channels and micropores that influence the motion of solutes in the biofilm) is dependent on the rate of internal mass transfer and, at the same time, the structure itself affects the internal "effective diffusivity". The latter may comprise molecular and turbulent diffusion, with or without convection/advection.

Biofilms can be seen as catalysts (living ones). As opposed to chemical catalysts, they are able to grow and modify their internal structure in order to adapt to the operating conditions. Data from a great number of authors has reported on how biofilm physical structure changes according to the hydrodynamics and composition of the external liquid (Vieira et al., 1993; Peyton, 1996; Hermanowicz, 1999; Melo and Vieira, 1999; Beyenal and Lewandowski, 2002; Wijeyekoon et al., 2004) and, as a consequence, how internal diffusivities are affected by external variables such as fluid velocity, flow regime, nature and concentration of substrates (Fan et al., 1990; Vieira et al., 1993; Bishop et al., 1995; Stewart, 1998; Beyenal et al., 1998; Beyenal and Lewandowski, 2000).

Research in the last decade showed us that most biofilms are predominantly heterogeneous matrices with clusters made of cells and polymers, randomly distributed along a three-dimensional architecture and surrounded by water channels that sometimes extend from the top to the bottom of the biofilm. In general, the water content of biofilms reaches $90-99 \%$ of the total wet mass. Therefore, why worry about internal mass transfer rates and why not consider them equal to the external mass transfer rates? The point is 
that the biofilm matrix is a kind of "bi-model pore structure" containing large macropores (water channels) between different cell-polymer aggregates and micro-pores inside these aggregates. In the macro-pores mass transfer by molecular diffusion can sometimes be enhanced by convection, but in the smaller pores diffusivity can be much slower due to the "compactness" (see next sub-section) of the cell-polymer mass.

It could be argued that in membrane attached biofilms (such as in membrane reactors and in some diffusion measurement apparatuses with porous membranes) substrates/nutrients would certainly tend to choose the large water channels (macro-pores) as wide "motorways" to travel across biofilms (by convection/advection) and would avoid crossing through the denser clusters. However, the reaction processes inside biofilms use up such molecules and create concentration gradients that are the real driving force for diffusion mass transfer through the cell-polymer clusters. Therefore, whatever the case, substrate molecules are forced to cross through complex paths and that is what potentially makes internal diffusion an important "limiting step" that often determines the overall biological activity of the biofilm.

Because of the heterogeneity of biofilms, properties may change drastically from one point to another inside the matrix and this applies also to the parameters that quantify mass transfer rates. Nevertheless, it is advisable, from a practical viewpoint, to obtain average values of such parameters to incorporate in engineering models. There are many data on average effective internal diffusivities measured by different authors, some of them (e.g. Beyenal and Lewandowski, 2000) having reached such values by first determining local diffusivities and then averaging them by reliable methodologies. The discussion here presented will be focused solely on the analysis of average internal diffusivities.

\section{Biofilm physical properties versus internal effective diffusivity}

A significant amount of literature has been published on the relation between internal mass transfer and the physical characteristics of the biofilms (Fan et al., 1990; Bishop et al., 1995; Stewart, 1998; Beyenal and Lewandowski, 2002; Stewart, 2003). The commonly accepted interpretation is that the effective diffusivity depends on the biofilm dry density (usually defined as the mass of dry biofilm per unit wet volume). Empirical correlations were presented that describe a decrease in diffusivity values with an increase in biofilm dry density (Fan et al., 1990). However, there are some cases where the correlations were not confirmed (e.g. Zhang et al., 1998; Casey et al., 2000 - see Table 1). The author of the present paper also came across such "deviations" when working with "well-fed" biofilms formed under laminar regime (quite low liquid velocities) without any substrate limitations (Garcia López et al., 2003). It can be argued that those few cases are not significant and could be the result of undetected inaccuracies.

But the question is more fundamental: what makes a given molecule move more or less quickly through the biological matrix? Is it the concentration of dry matter or the tortuosity (the "zig-zag" features) of the paths that the molecule has to follow? Both seem to be relevant and both contribute to the "compactness" of the biofilm. Let us look at the matrix from the viewpoint of the "travelling molecule". In fact, since biofilm composition is clearly dominated by its water content ( $90 \%$ or more), the differences in the amount of dry biomass from case to case become relatively unimportant for this molecule. Why should a molecule increase its transport velocity as a result of a $20 \%$ decrease in the dry density, when this corresponds only to a very small difference in the fraction of water in 


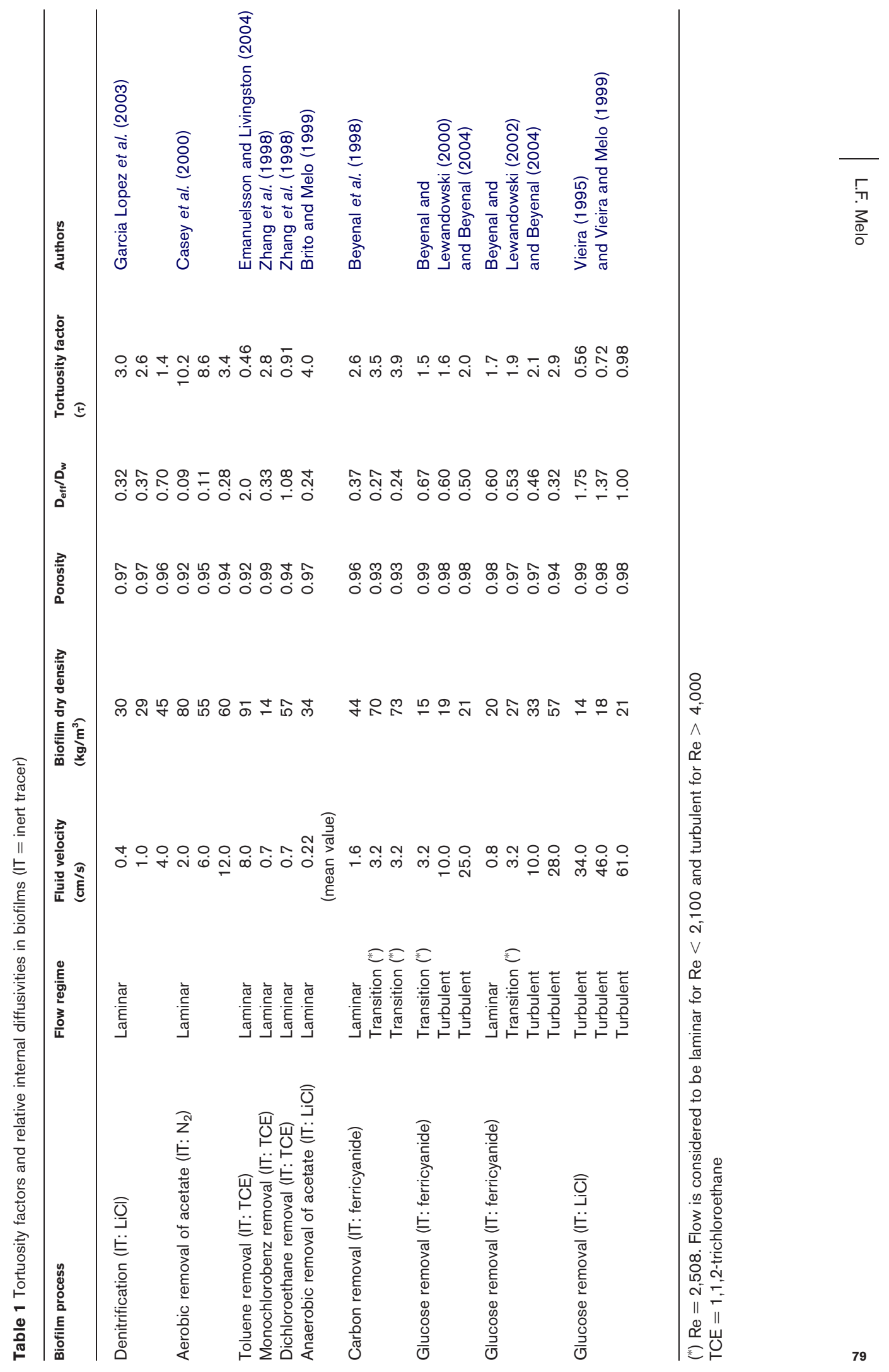


same in both cases and it should not result in significantly different diffusivities (or "travelling velocities").

It seems therefore clear that even biofilms with similar densities (mass of dry matter per unit wet volume) can display quite different diffusivity values (Table 1) depending on the tortuosity of the polymeric matrix. This is, in my view, the property that is more meaningful from a physical perspective, although biomass density cannot simply be ruled out as a relevant parameter. As a consequence, the tortuosity factor was chosen here to describe the physical features of biofilms that directly affect the average internal effective diffusivity.

Tortuosity has a simple geometrical meaning: it describes "the longer connecting path imposed by obstacles within porous solids relative to that for motion in unconstrained free space" (Zalc et al., 2004). Different definitions of "tortuosity factors" have been proposed and are commonly used in heterogeneous chemical catalysis. I will use the simplest one, based on the so-called "cylindrical model", which is easier to visualize. Using this definition of tortuosity factor $(\tau)$, the effective diffusivity inside the biofilm and the corresponding diffusivity in the bulk liquid are related by the following expression:

$\mathrm{D}_{\mathrm{eff}}=\frac{\mathrm{D}_{\mathrm{w}} \cdot \varepsilon}{\tau}$

where $\mathrm{D}_{\text {eff }}$ is the "average internal effective diffusivity" in the biofilm, $\mathrm{D}_{\mathrm{w}}$ is the molecular diffusivity in water and $\epsilon$ is the biofilm porosity. The equation shows that internal diffusion is not only dependent on the density of dry matter (directly related to the porosity $\epsilon)$, but also on the tortuosity $(\tau)$ of the matrix composed by that dry matter. It should be emphasized that other authors (Zhang and Bishop, 1994; Bishop et al., 1995) have already mentioned this parameter as a crucial one in interpreting diffusion in biofilms. However, design engineers are still waiting for practical ways of relating diffusivity in biofilms to reactor operating conditions. The present paper shows how the tortuosity concept fits easily in the existing data and presents illustrative examples of the relationships between tortuosity factors, density, internal diffusivities and fluid velocity.

\section{Density versus tortuosity}

Table 1 summarizes data from a variety of authors working with quite distinct biofilms formed by different microbial species, with different substrates and different hydrodynamics. The values of dry density (dry mass divided by wet volume of biofilm) were measured by the authors, except in the case of Lewandowski and co-workers who obtained them from the correlation published by Fan et al. (1990). When not explicitly reported by the authors, porosity was calculated from the measured dry density values by using (Eq. 2):

$\varepsilon=1-\frac{\rho_{d w}}{\rho_{d}}$

where $\rho_{\mathrm{dw}}$ is the dry mass per unit wet volume and $\rho_{\mathrm{d}}$ is the "true" density of dry biomass obtained by dividing the dry biofilm mass by the volume of the dry material (excluding the air in the voids). The density of microbial cells containing water is around $1,000 \mathrm{~kg} / \mathrm{m}^{3}$, whereas the density of dry cells may vary between 200 and $670 \mathrm{~kg} / \mathrm{m}^{3}$ (Hinson and Kocher, 1996). On the other hand, the density of EPS material is always higher than $1,000 \mathrm{~kg} / \mathrm{m}^{3}$. For example, xanthan true density is around $1,500 \mathrm{~kg} / \mathrm{m}^{3}$ (Talukdar et al., 1996); true density of the EPS material produced by Zoogloea ramigera is $1,300 \mathrm{~kg} / \mathrm{m}^{3}$ (Mueller et al., 1968). Although some authors have used $300 \mathrm{~kg} / \mathrm{m}^{3}$ for $\rho_{\mathrm{d}}$, it seems more acceptable to use a much higher value for this parameter, mainly when biofilms 
contain a significant fraction of EPS versus cell mass. The cases mentioned in Table 1 refer to flowing water with relatively high substrate concentrations (typical of wastewaters) and therefore one expects those biofilms to have a higher fraction of EPS in their dry mass. The approximate value of $1,000 \mathrm{~kg} / \mathrm{m}^{3}$ was therefore used here as a more reasonable compromise between the true densities of cells and of EPS material.

All the data in Table 1 were obtained in experiments with inert tracers, which avoids the uncertainties inherent to the use of mathematical model assumptions. The experimental data from Beyenal and Lewandowski (2000) shown in Table 1 were obtained with $150 \mathrm{ppm}$ of glucose; these authors reported other results with different concentrations, but with similar trends.

Figure 1 presents the diffusivity values from Table 1 as a function of biofilm density. The data is quite scattered and it shows that the biofilm dry density (dry mass per unit wet volume of the biofilm) may not be appropriate to interpret the diffusivity values. The values in Table 1 emphasize that in laminar flow higher densities may correspond either to higher or to lower diffusivities. On the other hand, under turbulent regime, an increase in biofilm density always corresponds to an increase in tortuosity and a decrease in internal diffusivity. Tortuosity factors lower than 1 may seem strange, but they simply confirm the existence of convection and turbulent diffusion inside the biofilm matrix. In such cases, $\mathrm{D}_{\text {eff }}$ is not really a molecular diffusivity but a parameter that encompasses all the possible mass transfer mechanisms in the biofilm.

If the relative average effective diffusivities $\left(D_{\text {eff }} / D_{w}\right)$ were plotted against the tortuosity factors, the line would obviously follow (Eq.1), that is, increased tortuosity leads always to decreased diffusivity. In the present case, since porosity is almost constant ranging between 0.92 and 0.99 , the curve would closely resemble a hyperbolic function. The important aspect, however, is that the concept of tortuosity relates more clearly to the mass transfer phenomenon than the traditional biofilm density parameter (note that the latter is included in the definition of the tortuosity factor). In principle, tortuosity describes the ratio between a straight line and a line with curves and zig-zags: for similar biofilm thicknesses, the higher the tortuosity, the longer will be the path that the molecules have to travel through the microbial layer and, therefore, the lower will be the effective diffusivity.

Figures 2 and 3 illustrate that the relationship between density and tortuosity is not always the same, that is, a more dense biofilm is not necessarily a more tortuous biofilm, particularly in laminar flow systems.

An even less predictable trend was obtained by Casey et al. (2000) in laminar flow, whereby the tortuosity factor first decreases and then increases sharply with the increase in biofilm dry density (Table 1).

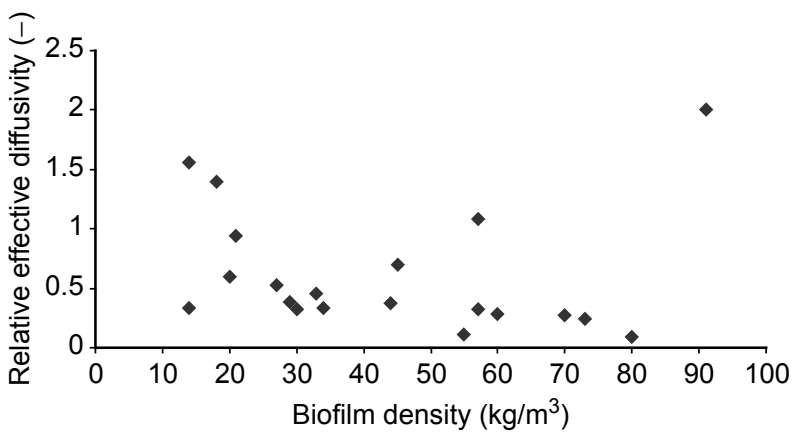

Figure 1 Average relative effective diffusivity as a function of biofilm density ( $\rho_{\mathrm{dw}}$, dry mass/wet volume) 


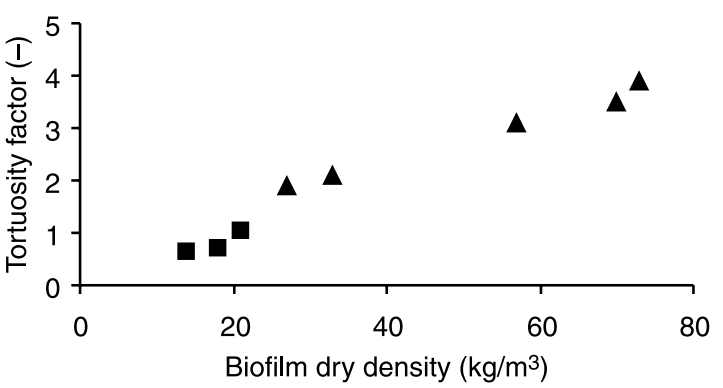

Figure 2 Tortuosity factors versus biofilm density (dry mass/wet volume) - turbulent and transition flow: - Vieira, 1995, Vieira and Melo, 1999; $\Delta$ - Beyenal et al., 1998, Beyenal and Lewandowski, 2002

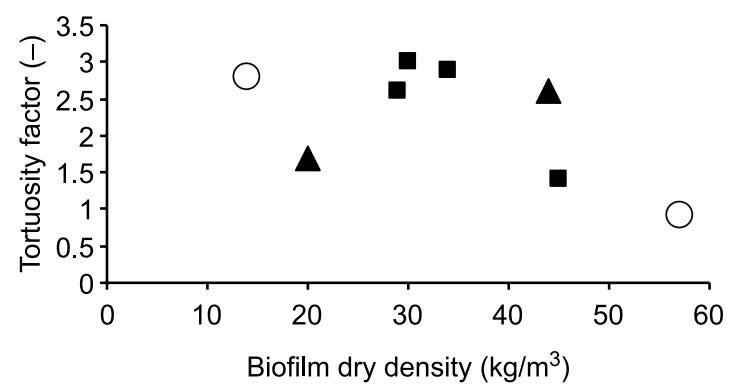

Figure 3 Tortuosity factors versus biofilm density (dry mass/wet volume) - laminar flow: $\mathbf{a}$ - Garcia-Lopez et al, 2003, Brito and Melo, 1999; $\mathbf{\Delta}$ - Beyenal et al., 1998, Beyenal and Lewandowski, 2002; O - Zhang et al., 1998

\section{Effect of fluid velocity on internal structure and mass transfer}

Figure 4 displays the effects of the fluid velocity on the relative effective diffusivity $\left(D_{\text {eff }}\right.$ $/ \mathrm{D}_{\mathrm{w}}$ ), as reported by three diferent research groups (Lewandowski and co-workers; Melo and co-workers and Casey et al.). Although the ranges of diffusivity values differ from group to group, the diffusivity seems to decrease (although only very slightly in the case of Lewandowski and co-workers) when the fluid velocity increases when the biofilms were formed under turbulent flow conditions. However, under laminar flow it is clear that the trends vary substantially: diffusivities may increase or decrease with the fluid velocity.

\section{Conclusions}

The paper reviews a significant quantity of diffusivity values measured by several authors with inert tracers. The usual dependency of the relative effective diffusivity on the biofilm density is not confirmed and does not seem an appropriate concept to explain the variability found in the experimental trends. Tortuosity is proposed here as a more relevant concept to describe mass transfer inside biofilm matrices, and also includes the effect of dry biomass density.

The work tries to assess the relationships between effective diffusivities and fluid velocity. The available data is scarce and the experimental conditions are not always clear in the published literature. In the case of biofilms formed under turbulent flow conditions, an increase in fluid velocity corresponds to a decrease in the diffusivity, although sometimes this decrease is very slight. However, in laminar flow situations, no common trend is found from research group to research group. 
(l)

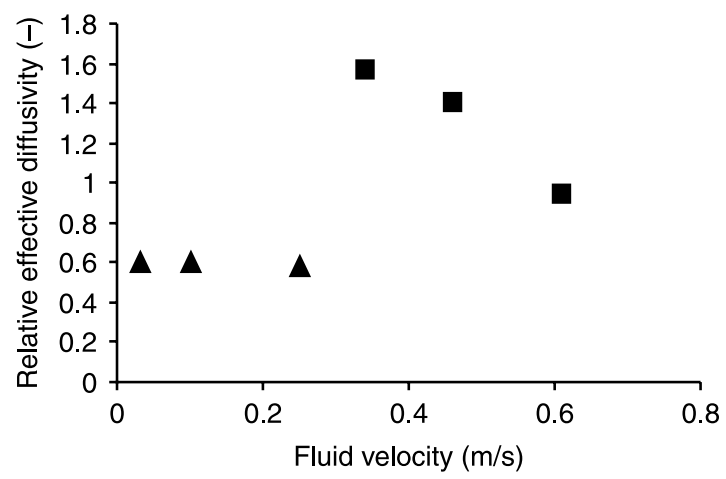

(II)

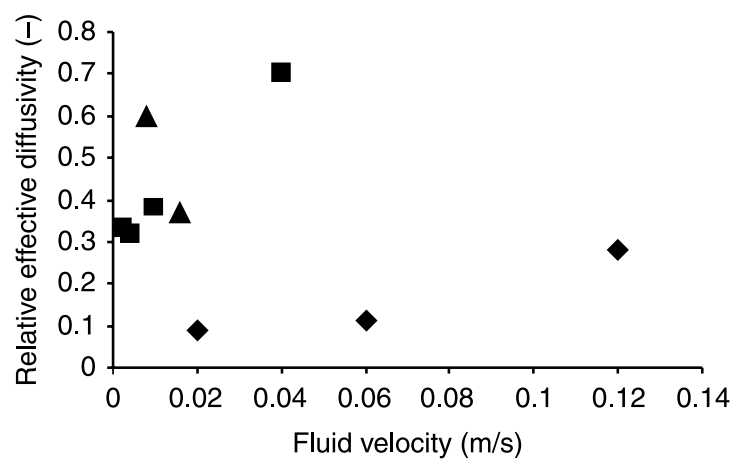

Figure 4 Effect of fluid velocity on the average relative effective diffusivities. I - Turbulent and transition flow: - Vieira, 1995, Vieira and Melo, 1999; $\mathbf{\Delta}$ - Beyenal and Lewandowski, 2000. II - Laminar flow: - Garcia-Lopez et al, 2003, Brito and Melo, 1999; $\mathbf{\Delta}$ - Beyenal et al., 1998; Beyenal and Lewandowski, 2002; - Casey et al., 2000.

It should be stressed that all biofilm parameters discussed in the paper (tortuosity, density, porosity, diffusivity, etc.) are presented as average values. These parameters vary from point to point within the biofilm matrix. For example, in large channels tortuosity is close to zero, whereas it will have high values inside more compact clusters composed by agglomerates of cells and EPS. In terms of average values, the conceptual physical model discussed in the paper can be applied to any type of spatial distribution of dry biomass in the biofilm, be it dispersed portions of biomass (cells plus EPS) in a hydrated environment or dispersed cells in a more homogeneous EPS network.

\section{References}

Beyenal, H. and Lewandowski, Z. (2000). Combined effect of substrate concentration and flow velocity on effective diffusivity in biofilms. Wat. Res., 34(2), 528-538.

Beyenal, H. and Lewandowski, Z. (2002). Internal and external mass transfer in biofilms grown at various flow velocities. Biotech. Prog., 18(1), 55-61.

Beyenal, H., Tanyolaç, A. and Lewandowski, Z. (1998). Measurement of local effective diffusivity in heterogeneous biofilms. Wat. Sci. Tech., 38(8-9), 171-178.

Beyenal, H. (2004). Personal communication.

Bishop, P.L., Zhang, T.C. and Fu, Y.-C. (1995). Effects of biofilm structure, microbial distributions and mass transport on biodegradation processes. Wat. Sci. Tech., 31(1), 143-152.

Brito, A.G. and Melo, L.F. (1999). Measurement of mass transfer coefficients in anaerobic biofilms. Wat. Res., 33(17), 3673-3678. 
Casey, E., Glennon, B. and Hamer, G. (2000). Biofilm development in a membrane-aerated biofilm reactor: effect of flow velocity on performance. Biotech. Bioeng., 67(4), 476-486.

Emanuelsson, E.A.C. and Livingston, A.G. (2004). Overcoming oxygen limitations in membrane-attached biofilms - an investigation of flux and diffusivity in an anoxic biofilm. Wat. Res., 38, 1530-1541.

Fan, L.-S., Leyva-Ramos, R., Wisecarver, K.D. and Zehner, B.J. (1990). Diffusion of phenol through a biofilm grown on activated carbon particles in a draft-tube three-phase fluidized bed bioreactor. Biotech. Bioeng., 35, 279-286.

Garcia López, L.A., Veiga, M.C., Nogueira, R., Aparicio, A. and Melo, L.F. (2003). A technique using a membrane flow cell to determine average mass transfer coefficients and tortuosity factors in biofilms. Wat. Sci. Tech., 47(5), 61-67.

Hermanowicz, S.W. (1999). Two-dimensional simulations of biofilm development: effects of external environmental conditions. Wat. Sci. Tech., 39, 107-114.

Hinson, R.K. and Kocher, W.M. (1996). Model for effective diffusivities in aerobic biofilms. J. Environ. Eng., 122(11), 1023-1030.

Melo, L.F. and Vieira, M.J. (1999). Physical stability and biological activity of biofilms formed under turbulent flow and low substrate concentration. Bioproc. Eng., 20, 363-368.

Mueller, J.A., Boyle, W.C. and Lightfoot, E.N. (1968). Oxygen diffusion through zoogloeal flocs. Biotech. Bioeng., 10, 331-358.

Peyton, B.M. (1996). Effects of shear stress and substrate loading rate on Pseudomonas aeruginosa biofilm thickness and density. Wat. Res., 30(1), 29-36.

Stewart, P. (1998). A review of experimental measurements of effective diffusive permeabilities and effective diffusion coefficients in biofilms. Biotech. Bioeng., 59(3), 261-272.

Stewart, P. (2003). Diffusion in biofilms. J. Bacteriol., 185, 1485-1491.

Talukdar, M.M., Vinckier, I., Moldenaers, P. and Kinget, R. (1996). Rheological characterization of xanthan gum and hydroxypropylmethyl cellulose with respect to controlled-release drug delivery. J. Pharm. Sci., 85(5), 537-540.

Vieira, M.J. (1995). Study of the formation of Pseudomonas fluorescens biofilms and of the effects associated to internal mass transfer and incorporation of kaolin particles. PhD Thesis, University of Minho (in Portuguese).

Vieira, M.J., Melo, L.F. and Pinheiro, M.M. (1993). Biofilm formation - hydrodynamic effects on internal diffusion and structure. Biofouling, 7, 67-80.

Vieira, M.J. and Melo, L.F. (1999). Intrinsic kinetics of biofilms formed under turbulent flow and low substrate concentration. Bioproc. Eng., 20, 369-375.

Wijeyekoon, S., Mino, T., Satoh, H. and Matsuo, T. (2004). Effects of substrate loading rate on biofilm structure. Wat. Res., 38, 2479-2488.

Zalc, J., Reyes, S.C. and Iglesia, E. (2004). The effects of diffusion mechanism and void structure on transport rates and tortuosity factors in complex porous structures. Chem. Eng. Sci., 59, 2947-2960.

Zhang, C.T. and Bishop, P.L. (1994). Density, porosity, and pore structure of biofilms. Wat. Res., 28(11), $2267-2277$.

Zhang, S.-F., Splendiani, A., Freitas dos Santos, L.M. and Livingston, A.G. (1998). Determination of pollutant diffusion coefficients in naturally formed biofilms using a single tube extractive membrane bioreactor. Biotech. Bioeng., 59(1), 80-89. 